\title{
Detection of Novel Red-Colored, Ultra-Weak Chemiluminescence from Carcinogens
}

\author{
Kazumi Osada, ${ }^{1, *}$ Yuji FuruKawa, ${ }^{1, * *}$ Michio KomaI, ${ }^{1}$ \\ Kohya Hishinuma, ${ }^{2}$ Mika Kimura, ${ }^{2}$ \\ Humio INABA, ${ }^{3}$ and Shuichi KIMURA ${ }^{1}$ \\ ${ }^{1}$ Laboratory of Nutrition, Faculty of Agriculture, and \\ ${ }^{3}$ Research Institute of Electrical Communication, \\ Tohoku University, Aoba-ku, Sendai 981, Japan \\ ${ }^{2}$ Biophoton Project, Research Development Corporation \\ of Japan, Aoba-ku, Sendai 980, Japan
}

(Received October 15, 1992)

\begin{abstract}
Summary We previously reported that benzo-(a)-pyrene (BP) and methylcholanthrene (MC) emitted blue-colored ultra-weak chemiluminescence (CL) during the metabolism in a post mitochondrial fraction system. In this present study, we used a single-photon counting system (SPC) equipped with a photomultiplier having a spectral response in the red-color region to detect the $\mathrm{CL}$ from $\mathrm{BP}, \mathrm{MC}$, and 3-nitrofluoranthene (3-NF). As a result, we discovered that a novel red-region CL was emitted immediately after initiation of the metabolic reaction in this system. In the case of BP and MC, each had a double phase CL emission; that is, they initially emitted red CL, which was detected by the SPC, and thereafter, blue $\mathrm{CL}$, which was detected with an OX-71 chemiluminescence analyzer. However, in the case of $3-\mathrm{NF}$, although strong red CL emission was recorded, no blue-colored $\mathrm{CL}$ appeared to be emitted. The emissions of red CL were increased in systems in which $\mathrm{D}_{2} \mathrm{O}$ was added (final concentration of 66\%), and decreased by the addition of $\beta$-carotene or $\alpha$-tocopherol. This result suggests that the main cause of CL was singlet oxygen.
\end{abstract}

Key Words: benzo-(a)-pyrene, methylcholanthrene, 3-nitrofluoranthene, chemiluminescence, singlet oxygen

The first investigation into the emission of chemiluminescence (CL) from

* Present address: OTC Product Development Division, Taisho Pharmaceutical Co., Ltd., Toshima-ku, Tokyo 171, Japan.

** To whom correspondence should be addressed. 
carcinogens was reported in 1974 by Seliger et al. [1]. In that report they said that there was an unidentified molecule in tobacco smoke that emitted a luminescence. They tried to clarify the emitting species by use of a microsomal fraction of rat liver, which is widely used as a model system for study of the metabolism of xenobiotics, and they suggested that the main emitting species was 7,8-diol-9, 10-dioxetane, one of the intermediate metabolites benzo-( $a$ )-pyrene (BP) [2]. They also indicated no evidence that singlet oxygen $\left({ }^{1} \mathrm{O}_{2}\right)$ was produced in this system [3]. Another study of chemiluminescence, using a microsomal fraction system, concentrated on aflatoxin B1 and 9,10-dimethyl-1,2-benzanthracene [4], but no comment was made about any correlation between $\mathrm{CL}$ and singlet oxygen. Recently we reported that a blue-colored, ultra-weak CL was detected not only from BP, but also from 3-methylcholanthrene (MC) in a post-mitochondrial fraction, which is a crude type of microsomal fraction widely used in the Ames test, and that these CLs were emitted from intermediate metabolites that were formed from $\mathrm{BP}$ and $\mathrm{MC}$ [5].

During this present study, we observed red-colored CL from BP, MC, and 3-nitrofluoranthene (3-NF), all of which are well-known air pollutants, and discovered that one of the emitting species could be singlet oxygen.

\section{MATERIALS AND METHODS}

Chemicals. The BP, MC, 3-NF, $\alpha$-tocopherol, and $\beta$-carotene used in this experiment were analytical grade and purchased from Wako Pure Chemical Industries Co., Osaka. Phosphatidylcholine (type-III-E), cholesterol, and deuterium oxide were purchased from Sigma Chemical Co., St. Louis, MO. All organic solvents used in this study were analytical grade.

Preparation of $S-9$ mix. The S-9 mix was prepared by a slight modification of the method used by Yahagi et al. [6]. Previous to homogenization, livers from decapitated male rats $(100 \mathrm{~g}$ weight $)$ were perfused through the portal vein with ice-cold $\mathrm{KCl}(150 \mathrm{~mm})$. The isolated livers were homogenized with 3 volumes of $\mathrm{KCl}(150 \mathrm{mM})$ and centrifuged at $9,000 \times \mathrm{g}$. To the resulting supernatant, NADPH was added at the concentration of $4 \mu \mathrm{mol} / \mathrm{ml}$ as a co-factor. The mixture thus obtained was designated S-9 mix.

Preparation of lecithin-cholesterol vesicles. The lecithin-cholesterol vesicles were prepared by the Batzri and Korn method [7] and were used as the mutagen carrier in this monitoring system. Two-hundred and twenty nanomoles of BP, MC or $3-\mathrm{NF}$ was mixed with $900 \mathrm{nmol}$ of egg lecithin and $150 \mathrm{nmol}$ of cholesterol and injected into a $39 \mathrm{~mm}$ sodium phosphate buffer $(5 \mathrm{ml})$. The mutagens thus set in lecithin-cholesterol vesicles were added to separate S-9 mix systems.

Measurement of ultra-weak chemiluminescence. A synchronous, singlephoton-counting apparatus, a chemiluminescence Analyzer OX-71 manufactured by Tohoku Electronic Industries Co., Sendai, was used and equipped with a Hamamatsu R878 photomultiplier having a special response range of $300-650 \mathrm{~nm}$ 
(sensitivity maximum at $400 \mathrm{~nm}$ ). This analyzer was used to detect blue-colored CLs.

To detect red-colored CL, we used a single-photon counting system (SPC) originally constructed by the Biophoton Project Research Development Corporation of Japan using a commercial photomultiplier tube (R1333, special response range of 420-800 nm, sensitivity maximum at $600 \mathrm{~nm}$; Hamamatsu Photonics Co., Ltd., Shizuoka, Japan) and a photon counter (SR-400, Stanford Research System Inc., Palo Alto, CA).

The same reaction conditions were used here as were used in our previous investigation [5]. In short, the sample solution consisting of $2 \mathrm{ml}$ of S-9 mix and $1 \mathrm{ml}$ of polyaromatic hydrocarbon set in lecithin-cholesterol vesicles was placed on a stainless-steel plate (OX-71) or glass dish (SPC), and monitored at $37^{\circ} \mathrm{C}$ for 20-30 min. The emission intensity was expressed in terms of average counts per $30 \mathrm{~s}$.

Simple treatment of $S-9$ mix system with $\alpha$-tocopherol, $\beta$-carotene, and $\mathrm{D}_{2} \mathrm{O}$. $\mathrm{D}_{2} \mathrm{O}$ prolongs the lifespan of singlet oxygen [8], and $\alpha$-tocopherol and $\beta$-carotene are well known as quenchers of singlet oxygen $[9,10]$. These compounds were used to detect whether or not singlet oxygen is the emitting species of the redcolored CL. $\beta$-Carotene $(100 \mathrm{nmol})$ or $\alpha$-tocopherol $(380 \mathrm{nmol})$ were each added to a separate $\mathrm{S}-9$ mix system. $\mathrm{D}_{2} \mathrm{O}$ was mixed with a third $\mathrm{S}-9$ mix system to produce a final concentration of $66 \% \mathrm{D}_{2} \mathrm{O}$.

\section{RESULTS}

Detection of blue- and red-colored CLs from benzo(a)pyrene, methylcholanthrene, and 3-nitrafluoranthene

In the case of each of $\mathrm{BP}, \mathrm{MC}$, and 3-NF, red-colored $\mathrm{CL}$, though weak, was detected after $10 \mathrm{~min}$ from the initiation phase of the enzyme reaction in the S-9 mix system. However, the $\mathrm{CL}$ of each appeared to be at its maximum emission stage after 3-5 min.

In the case of $\mathrm{BP}$ and $\mathrm{MC}$, the blue-colored $\mathrm{CL}$ was displayed in a broad peak of emission after the decay of the red CL (Fig. 1 a, b). Although both of the CLs from $\mathrm{MC}$ were weaker than those from $\mathrm{BP}$, they had a clear double-phase pattern similar to that of BP. On the other hand, 3-NF only emitted strong red CL (Fig. $1 \mathrm{c})$.

Effect of $\beta$-carotene, $\alpha$-tocopherol, and $D_{2} \mathrm{O}$ on intensity of red-colored $C L$ from $B P$ and $3-N F$

In an attempt to ascertain whether the emitting species is singlet oxygen or not, we attempted to detect whether any change in the intensity of the red-colored $\mathrm{CL}$ from BP and MC would result from the addition of singlet-oxygen scavengers, namely, $\beta$-carotene and $\alpha$-tocopherol, or from the addition of $\mathrm{D}_{2} \mathrm{O}$, which extends the lifespan of singlet oxygen about ten-fold. In the case of both BP and 3-NF, 

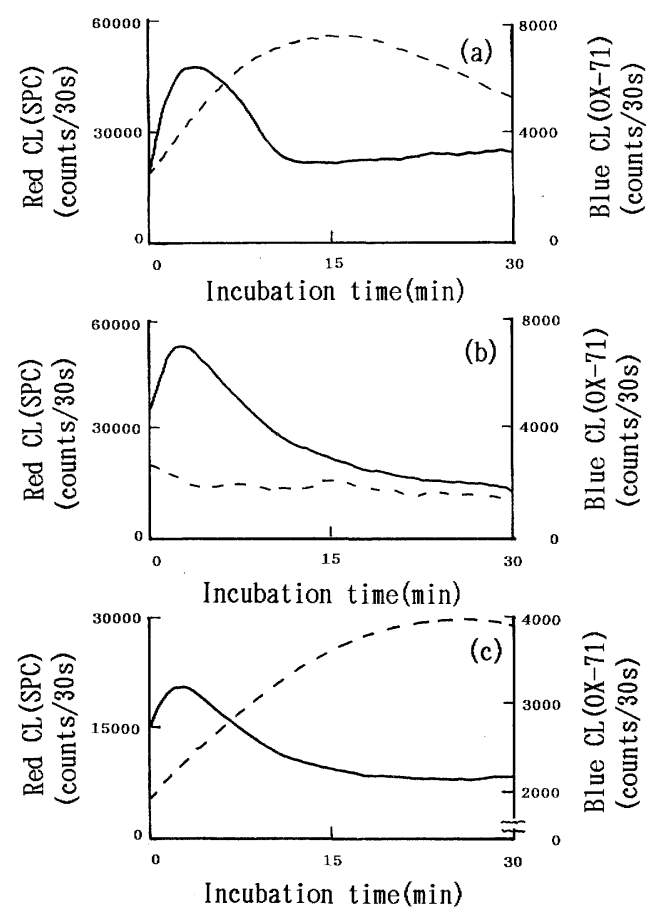

Fig. 1. Change in chemiluminescence intensity from $\mathrm{BP}(\mathrm{a}), 3-\mathrm{NF}(\mathrm{b})$, and $\mathrm{MC}(\mathrm{c})$ as a function of time. SPC has a sensitivity maximum at $600 \mathrm{~nm}$ (Red CL); and OX-71, at $400 \mathrm{~nm}($ Blue $\mathrm{CL}) . \_$, CL intensity measured with SPC; --.-, CL intensity measured with OX-71.

$\beta$-carotene and $\alpha$-tocopherol effectively quenched the CLs. In particular, $\alpha$ tocopherol was the more effective. The addition of $\mathrm{D}_{2} \mathrm{O}$ (final concentration of $66 \%$ ) to the S-9 mix system significantly amplified the intensities and prolonged the emission times of the CLs from BP and 3-NF (Fig. 2 a, b).

\section{DISCUSSION}

By employing two types of photomultipliers, Seliger et al. [3] detected the red and blue CLs emitted by BP-7,8-diol (a proximate carcinogen derived from benzo- $(a)$-pyrene) and by cis- and trans-methoxyvinylpyrene (an analogue of BP-7,8-diol) in a microsome system. In both cases, the red CL and the blue CL showed similar kinetics. Seliger suggested that there was no evidence that singlet oxygen was produced as the emitting species, during the metabolic reaction in the microsomal system.

In our study, we used the post-mitochondrial system ( $\mathrm{S}-9 \mathrm{mix}$ ). This may provide one reason as to why we could observe a difference in the behavior of the red CLs which appeared rapidly in the initiation phase and the blue CLs which 


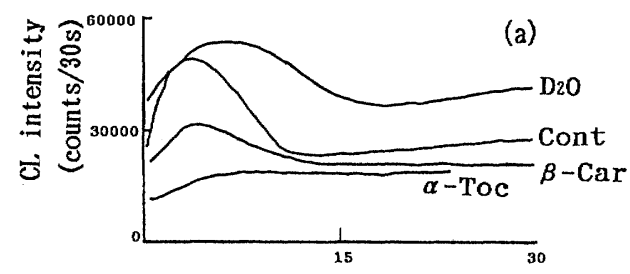

Incubation time(min)

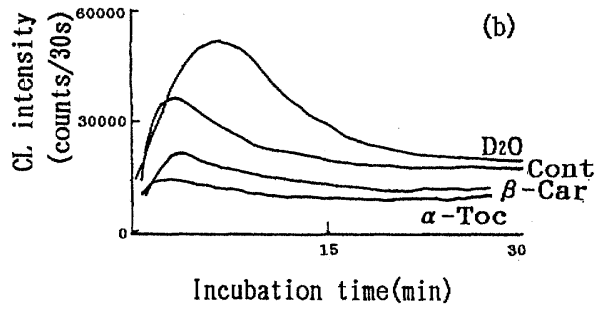

Fig. 2. Effects of singlet-oxygen quenchers and deuterium oxide on intensity of CL from $\mathrm{BP}(\mathrm{a})$ and 3-NF(b). Experimental conditions are given in Materials and Methods. Results are expressed as the single measurement of chemiluminescence representative of one of the five experiments conducted.

were emitted more slowly than the red ones. Our results clearly demonstrate that there are two types of CL, namely, red CL, emitted in the initiation phase, and blue CL, which appeared more slowly.

The CLs were amplified on the addition of $\mathrm{D}_{2} \mathrm{O}$ to this system, which substance prolongs the lifetime of singlet oxygen, and were reduced on the addition of $\beta$-carotene or $\alpha$-tocopherol, known quenchers of singlet oxygen. Consequently, our results suggest that the major part of the emitted red CL from $\mathrm{BP}$ and $3-\mathrm{NF}$ is due to singlet oxygen. In both cases, singlet oxygen appeared in the initiation phase of metabolism of $\mathrm{BP}$ and 3-NF in the post-mitochondrial supernatant system.

On the other hand, we noticed that in the case of $3-\mathrm{NF}$, only red $\mathrm{CL}$ was emitted. This result suggests that $3-\mathrm{NF}$ has no metabolites emitting blue CL but that singlet oxygen is produced during its metabolism.

We could not detect the emission spectrum of the CLs in detail because they did not have a plateau phase and the maximum emission intensity was not constant. Since it is one of the specific markers of the appearance of singlet oxygen $[11,12]$, we tried to detect $1,268-\mathrm{nm}$ infrared chemiluminescence, by using a single photoelectron counting system originally constructed by Biophoton Project members. However, we could not detect the 1,268-nm emission. The singlet oxygen that appears in the metabolism of BP, MC, and 3-NF may be too low in concentration to allow detection of this CL even with this new type of CL analyzer.

Through further improvements in this type of analyzer, it should become even more certain that singlet oxygen appears in the initiation phase of the metabolism 
of BP, MC, and 3-NF and potentially in that of other chemicals as well.

\section{REFERENCES}

1. Seliger, H.H., Biggley, W.H., and Hamman, J.P. (1974): Long-lived chemiluminescence in cigarette smoke. Science, 119, 253-256.

2. Hamman, J.P., Seliger, H.H., and Posner, G.H. (1981): Specificity of chemiluminescence in the metabolism of benzo-(a)-pyrene to its carcinogenic diol epoxide. Proc. Natl. Acad. Sci. U.S.A., 78, 940-942.

3. Tompson, A., Biggley, W.H., Posner, G.H., Lever, J.R., and Seliger, H.H. (1986): Microsomal chemiluminescence of benzo- $(a)$-pyrene-7,8-dihydrodiol and its synthetic analogues trans- and cis-1-methoxyvinyl pyrene. Biochim. Biophys. Acta, 882, 210-219.

4. Emerole, G.O., and Dixon, R.L. (1980): Evaluation of chemiluminescence generation during microsomal metabolism of some carcinogens. Cancer Res., 40, 2002-2005.

5. Osada, K., Furukawa, Y., Komai, M., and Kimura, S. (1990): Detection of ultra weak chemiluminescence from benzo(a)pyrene and 3-methylcholanthrene. J. Clin. Biochem. Nutr., 8, 185-192.

6. Yahagi, T., Nagao, M., Seino, Y., Matsushima, T., Sugimura, T., and Okada, M. (1977): Mutagenicities of N-nitrosamines on salmonella. Mutat. Res., 48, 121-130.

7. Batzri, S., and Korn, E.D. (1973): Single bilayer liposomes prepared without sonication. Biochim. Biophys. Acta, 298, 1015-1019.

8. Merkel, P.B., and Kearuns, D.R. (1972): Radiationless decay of singlet molecular oxygen in solution. An experimental and theoretical study of electronic-to-vibrational energy transfer. J. Am. Chem. Soc., 94, 7244-7253.

9. Berton, J., and Mathis, P. (1970): Mise en evidence de l'etat triplet de la chlorophylle dans des lamelles chloroplastiques. C.R. Acad. Sci. Serd, 271, 1094-1097.

10. Foote, C.S., Chang, T.Y., and Geller, G.G. (1974): Chemistry of singlet oxygen-xvIII. Rates of reaction and quenching of $\alpha$-tocopherol and singlet oxygen. Photochem. Photobiol., 20, 511-513.

11. Kanofsky, J.R. (1983): Singlet oxygen production by lactoperoxidase. J. Biol. Chem., 258, 5991-5993.

12. Kanofsky, J.R. (1984): Singlet oxygen production by chloroperoxidase-hydrogen peroxidehalide systems. J. Biol. Chem., 259, 5596-5600. 